\title{
Isomerism and decoherence
}

\author{
Sebastian Fortin ${ }^{1}$ - Olimpia Lombardi ${ }^{2}$ - Juan Camilo Martínez González ${ }^{1 *}$ \\ ${ }^{1}$ CONICET, Universidad de Buenos Aires. \\ ${ }^{2}$ CONICET, Universidad de Buenos Aires, Theiss Research.
}

\section{1.- Introduction}

Since the advent of quantum mechanics and its application to chemical systems, reduction became a regulative idea in the accounts of the relationship between physics and chemistry. In the famous introductory paragraph of his article of 1929, Paul Dirac described the central idea of what later would be known as quantum chemistry: "The underlying physical laws necessary for the mathematical theory of a large part of physics and the whole of chemistry are thus completely known, and the difficulty is only that the exact application of these equations leads to equations much too complicated to be soluble. It therefore becomes desirable that approximate practical methods of applying quantum mechanics should be developed, which can lead to an explanation of the main features of complex atomic systems without too much computation" (Dirac 1929, p. 714). The approximate methods referred to by Dirac, which are the core of quantum chemistry, constitute the field in which the discussion about reduction acquires particular relevance. In fact, the strategies of approximation that make possible the description of chemical phenomena in quantum terms, such as the Born-Oppenheimer approximation or the models of Valence Bond and Molecular Orbital, do not satisfy the conditions of Nagelian reduction (Nagel 1961): they not only establish loose and non-continuous connections between chemistry and physics, but also introduce assumptions that are incompatible with quantum mechanics itself.

In this article we will address the problem of the relation between chemistry and physics with the focus on the problem of optical isomerism and the so called "Hund's paradox." For this purpose we will begin by framing the problem in the context of the discussions about the possibility of explaining molecular structure in quantum terms. Then, we will consider the particular case of optical isomers, stressing that quantum mechanics cannot account for the difference between isomers of opposite chirality, and that this fact does not depend on

\footnotetext{
* This work is fully collaborative: the order of the names does not mean priority.
} 
idealizations or approximations but is the consequence of the peculiar form of the Hamiltonian of the chiral molecules. This will lead us to introduce Hund's paradox, which points to the difficulty to give a quantum explanation to chirality. On this basis, next we will describe the solution proposed from the perspective of the theory of quantum decoherence. Finally, we will question this solution in the light of a precise interpretation of the phenomenon of decoherence. Our closing suggestion will be that a satisfactory solution to Hund's paradox can only be reached by means of an adequate interpretation of quantum mechanics.

\section{2.- The problem of molecular structure}

The approximate methods used in quantum chemistry play a leading role in deciding about the referent of chemical concepts, such as orbital or chemical bond (Hettema 2009). In this context, the concept of molecular structure is particularly relevant, since considered as "the central dogma of molecular science" (Woolley 1978, p. 1074): molecular structure is the main factor in the explanation of reactivity. The problem consists in the fact that the concept seems to find no place in the theoretical framework of quantum mechanics, since it appeals to classical notions such as the position of the atomic nuclei or the individuality of electrons, both ideas strongly challenged in the quantum context. Although this problem has attracted the attention of several authors, the discussion is far from settled: the opinions about the link between quantum concepts and the notion of molecular structure diverge markedly.

Guy Woolley $(1976,1978,1982,1998)$ is one of the first authors in dealing with the implications of the approximation Born-Oppenheimer and the quantum theory of molecular structure in a specifically chemical context. According to the author, by means of the description of a molecule from "first principles", that is, as an isolated dynamical system composed of nuclei and electrons that interact through electromagnetic forces, "one cannot even calculate the most important parameters in chemistry, namely, those that describe the molecular structure" (Woolley 1978, p. 1074). Woolley considers that the impossibility of determining the geometry of a molecule by means of quantum mechanics is a proof of the fact that molecular shape is not an intrinsic property of molecules, but it is only a "powerful and illuminating metaphor" (Woolley 1982, p. 4). 
In the field of the philosophy of chemistry, Robin Hendry $(2004,2008,2010)$ has largely treated the issue of molecular structure in the context of the problem of reduction. According to the author, reduction has to be discussed in the ontological domain, because the impossibility of epistemological (inter-theoretical) reduction of chemistry to physics is already indisputable: even the reductionist knows that "the exact equations are insoluble, [...] the semi-empirical models are approximations to rigorous treatments, standing in for them in explanations of specialscience phenomena." (Hendry 2010, p. 184). On this basis, Hendry considers that the relationship between quantum mechanics and molecular chemistry, embodied in the way in which the molecular structure of a system of several electrons and one nucleus is explained, must be conceived in terms of emergence.

Whereas Woolley and Hendry insist on the difficulties of explaining molecular structure in exclusively quantum terms, Hinne Hettema (2012, Chap. 3) adopts a position explicitly reductionist by claiming that the inter-theoretic relationship between molecular chemistry and quantum mechanics has not been properly formulated in present-day philosophy of chemistry. For the author, the problem lies, at least partially, in the scarce impact that the recent developments in quantum chemistry have had in the present day philosophy of chemistry. Among these developments, he particularly points out the "Quantum Theory of Atoms in Molecules" (QTAiM, Bader 1994), which would make possible to reconstruct the structure of a molecule by means of the topology of its electronic density. According to Hettema, this approach fulfills the conditions required by the traditional model of reduction (Nagel 1961).

Interestingly, during the last years also Woolley has begun to soften his view. In particular, in his recent works with Brian Sutcliffe (Sutcliffe and Woolley 2011, 2012), the impossibility of deriving molecular structure from quantum mechanics is not conceived as the result of a conceptual obstacle, but as the consequence of our partial knowledge of the molecular systems in the theoretical framework of quantum mechanics: "We have never claimed that molecular structure cannot be reconciled with or reduced to quantum mechanics, or that there is something 'alien' about it; our claim is much more modest. We do not know how to make the connection." (Sutcliffe and Woolley 2011, p. 94).

A central element in the discussion about molecular structure is the role played by the Born-Oppenheimer approximation, whose fundamental premise is the possibility of decomposing 
the Hamiltonian of the molecule into its electronic and its nuclear components. This move relies on assuming the nuclei as classical-like particles at rest in a definite position: on this basis, the terms of kinetic energy associated to the nuclear motion are removed (clamped nuclei assumption). In the Hamiltonian resulting from that assumption, the positions of the nuclei are what describe the molecular structure. In turn, the potential energy surface (PES) that affects the electrons of the molecule can be calculated by means of this Hamiltonian. Nevertheless, from the viewpoint of reduction, the Born-Oppenheimer approximation faces some difficulties. First, it introduces the molecular structure into the quantum description from the very beginning, when the positions of the nuclei are established with the appeal to classical geometric considerations. Second, the assumption of the nuclei at rest in fixed spatial positions is in contradiction with the Heisenberg principle, which prevents quantum systems from having definite values of position and velocity simultaneously (see Lombardi and Castagnino 2010).

As indicated above, the discussion around the nature of molecular structure, often related with the interpretation of the Born-Oppenheimer approximation, is far from settled. However, there is a specific problem related with chemical structure that has been gaining ground in the foundations of chemistry as a relevant point in the debate about the relationship between molecular chemistry and quantum mechanics. This is the problem of isomerism, and in this case the difficulties are independent from the assumptions involved in the Born-Oppenheimer approximation.

\section{3.- Isomerism and optical activity}

As it is well known, isomerism -the existence of molecules that have the same numbers of the same kinds of atoms (and hence the same formula) but differ in chemical and physical properties- is a phenomenon particularly relevant in chemistry to explain the difference in the behavior of substances with the same composition. Here we will restrict our attention to optical isomers: the members of a pair of optical isomers are non-superposable mirror images. The property that distinguishes the members of the pair is chirality. Chiral molecules have an important function in the enzymatic reactions of biological systems: many pharmacological drugs are chiral, and generally only one of the members of the pair exhibits biological activity. 
Let us recall that the peculiarity of optical isomers of a same compound is that they share almost all their chemical and physical properties. Physically, they only differ in their interaction with polarized light: they rotate the plane of polarization of plane-polarized light. Depending on the direction of the rotation, dextro-rotation (clockwise) or levo-rotation (anticlockwise), optical isomers are called $D$ or $L$ optical isomers. When a compound contains equal quantity of $D$ and $L$ optical isomers, it is called racemic mixture; in this case, the net rotation is zero. In general, an optically active substance tends to become an optically inactive mixture of equal amounts of dextro-rotatory and levo-rotatory isomers: this process is called racemization.

When a molecule is described from a quantum perspective, the Coulombian Hamiltonian only depends on the distances between the particles composing the molecule; in particular, if only the atomic nuclei are considered, the Hamiltonian depends exclusively on the inter-nuclear distances. In the case of structural isomers -molecules with the same molecular formula but different in the structure or bonding-, the difference between them is manifest in the Hamiltonian itself and, with it, in their energies. But in the case of optical isomerism, all the inter-atomic distances are the same for the two members of the pair of isomers and, as a consequence, the Hamiltonian is exactly the same for both. This means that quantum mechanics supplies the same description for two structures that can effectively be distinguished in practice through their optical activity.

It is very important to stress that the problem of optical isomerism is completely independent of the Born-Oppenheimer approximation. Let us suppose, for instance, that we could count with the exact Hamiltonian $\hat{H}$ (with no approximation) of a molecule of alanine $\mathrm{C}_{3} \mathrm{H}_{7} \mathrm{NO}_{2}$, which includes three nuclei of carbon, one of nitrogen, and two of oxygen, plus all its electrons (Figure 1)

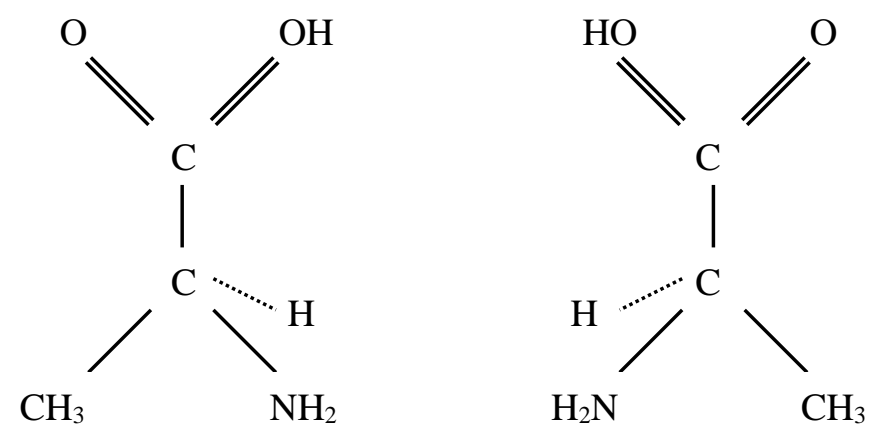

Figure 1: Optical isomers of alanine 
Even if we cannot write down this Hamiltonian due to its complexity, we know that it only depends on the distance of the component particles and, therefore, not even the exact Hamiltonian can account for the difference between the $D$-alanine and the $L$-alanine. As Sutcliffe and Woolley claim: "Clearly then, an eigenstate of $H$ does not correspond to a classical molecule with structure! That observation begs the question: what are the equations that determine the quantum state of molecules? Beyond the BO [Born-Oppenheimer] approximation we have no idea." (Sutcliffe and Woolley 2012, p. 416; emphasis in the original). In short, the problem of the quantum mechanical distinction of optical isomers of the same substance is beyond the BornOppenheimer approximation and its underlying assumptions. It is a problem that points to a deep difficulty in the attempts to account for molecular chemistry in quantum terms.

\section{4.- Optical isomerism and Hund's paradox}

The problem embodied in optical isomerism and its relations with molecular structure was already suggested by Friedrich Hund, pioneer in the development of quantum chemistry. Hund's paradox can be understood in two versions. In its first version, due to Hund himself (1927), it can be expressed as follows: given that the chiral states are not eigenstates of the Hamiltonian (since the Hamiltonian is parity invariant), and none of them corresponds to the basal state, $¿$ why certain chiral molecules display an optical activity that is stable in time, associated to a well-defined chiral state, and they are not in a superposition of the two possible chiral states? During the last decades, Hund's paradox was formulated in a slightly stronger version (Berlin, Burin and Goldanskii 1996): ¿why chiral molecules have a definite chirality? Below we will face the problem in precise formal terms.

Let us consider a chiral molecule from the perspective of quantum mechanics. For this purpose we have to construct the Hamiltonian of the complete molecule, taking into account all its components (nuclei and electrons) and their interactions. Let us suppose that the molecule consists of $A$ atomic nuclei, and each nucleus has an associated momentum operator $\hat{p}_{g}$, a mass $m_{g}$ and an atomic number $Z_{g}$, with $g=1,2, \ldots, A$. Since the components are charged particles, the interactions between them are Coulombian interactions: each particle interacts with all the rest, and the intensity of the interaction depends on the charge of each particle and the distances between them. The full Hamiltonian for this molecular system is (Szabo and Ostlund 1996): 


$$
\hat{H}=\sum_{g}^{A} \frac{\hat{p}_{g}^{2}}{2 m_{g}}+e^{2} \sum_{g<h}^{A} \frac{Z_{g} Z_{h}}{2 m_{g}}+\sum_{i}^{N}\left(\frac{\hat{p}_{i}^{2}}{2 m_{e}}-e^{2} \sum_{g}^{A} \frac{Z_{g}}{r_{i g}}\right)+e^{2} \sum_{i<j}^{N} \frac{1}{r_{i j}}
$$

where $e$ and $m_{e}$ are the charge and the mass of the $N$ electrons respectively, $\hat{p}_{i}$ is the momentum operator of the electron $i$, with $i, j=1,2, \ldots, N, r_{i j}$ is the "distance" between the electron $i$ and the electron $j$, and $r_{i g}$ is the "distance" between the electron $i$ and the nucleus $g$. Since the Coulombian interaction only depends on the distance between the interacting particles, it is symmetric under spatial reflection; therefore, the Hamiltonian commutes with the parity operator $\hat{P}$ :

$$
[\hat{P}, \hat{H}]=0
$$

This means that the eigenstates of the Hamiltonian have definite parity. Moreover, this feature is preserved during the time evolution of the system, because the parity operator commutes with the Hamiltonian and, as a consequence, is a constant of motion.

With these elements, Hund's paradox can be expressed in formal terms:

1. On the one hand, the eigenstates $\left|\omega_{n}\right\rangle$ of the Hamiltonian of this molecule have parity symmetry:

$$
\hat{P}\left|\omega_{n}\right\rangle= \pm\left|\omega_{n}\right\rangle
$$

This means that the geometry of the state $\left|\omega_{n}\right\rangle$ is invariant under space reflections. Therefore, the ground state $\left|\omega_{0}\right\rangle$, which is the state that quantum chemistry assigns to molecules, also has this symmetry. However, on the basis of experimental data it is possible to know that the states of optical isomers do not have this symmetry. In fact, if the two isomers corresponding to the two chiral states are represented by the quantum states $|L\rangle$ and $|R\rangle$, each isomer is the mirror image of the other, that is:

$$
\begin{aligned}
& \hat{P}|L\rangle=|R\rangle \\
& \hat{P}|R\rangle=|L\rangle
\end{aligned}
$$

Therefore, the states $|L\rangle$ and $|R\rangle$, as observed in the laboratory, cannot be eigenstates of the Hamiltonian.

\footnotetext{
${ }^{1}$ Although, strictly speaking, in quantum mechanics there is no distance between particles because in general they do not possess a definite position, the difference $\left|\bar{r}_{i}-\bar{r}_{j}\right|$ is usually called "distance between particles", where $\bar{r}_{i}$ and $\bar{r}_{j}$ are the coordinates of each electron in the position representation.
} 
2. On the other hand, the expression (2) shows that the parity symmetry is preserved through all the time evolution: $P$ is a constant of motion. This has the following consequence: if the initial state $\left|\varphi_{0}\right\rangle$ of the molecule has a definite parity, the state will preserve it at all later times:

$$
\hat{P}\left|\varphi_{0}\right\rangle=\left|\varphi_{0}\right\rangle \Rightarrow \hat{P}|\varphi(t)\rangle=|\varphi(t)\rangle
$$

Then, the process of racemization is very difficult to be explained in quantum terms, since it would imply the conversion of a dextro-rotating molecule into a levo-rotating molecule, or vice versa.

Once it is concluded that the states $|L\rangle$ and $|R\rangle$ are not eigenstates of the Hamiltonian, it is necessary to explain why those states are observed in the laboratory. One strategy consists in maintaining the Coulombian Hamiltonian, identifying the states $|L\rangle$ and $|R\rangle$ as superpositions of the eigenstates of the Hamiltonian, and then supplying a reason why the molecule does not decay to the ground state, eigenstate of the Hamiltonian. This is Hund's strategy, which will be considered below in formal terms.

The Coulombian Hamiltonian has eigenstates $\left|\omega_{n}\right\rangle$ with definite parity: the even levels have even parity, and the odd levels have odd parity. For instance, the ground state is symmetric:

$$
\hat{P}\left|\omega_{0}\right\rangle=+\left|\omega_{0}\right\rangle
$$

By contrast, the first excited state is anti-symmetric:

$$
\hat{P}\left|\omega_{1}\right\rangle=-\left|\omega_{1}\right\rangle
$$

These two eigenstates of the Hamiltonian are sufficient to construct a pair of chiral states $|L\rangle$ and $|R\rangle$ :

$$
\begin{aligned}
& |L\rangle=\frac{1}{\sqrt{2}}\left(\left|\omega_{0}\right\rangle+\left|\omega_{1}\right\rangle\right) \\
& |R\rangle=\frac{1}{\sqrt{2}}\left(\left|\omega_{0}\right\rangle-\left|\omega_{1}\right\rangle\right)
\end{aligned}
$$

These two states satisfy the relations of the expression (4) and, therefore, they can represent the states of the members of a pair of optical isomers.

By focusing only on the minimal elements that describe the situation, Hund poses the problem in a simpler system, with the advantage of the graphical visualization of the argument. 
Let us consider a quantum system with a potential $V(x)$ with mirror reflection symmetry, such as that of Figure 2. In this case, the fact that the potential has two minima leads to suppose that the system will tend to "move" toward one of the minima and, therefore, the stable states of the system are "at rest on the left" or "at rest on the right." From a classical viewpoint this is correct, but it is not correct in the quantum theoretical context. In quantum mechanics, the stable states of a system are the eigenstates of its Hamiltonian, and they always have the same symmetry of the Hamiltonian.

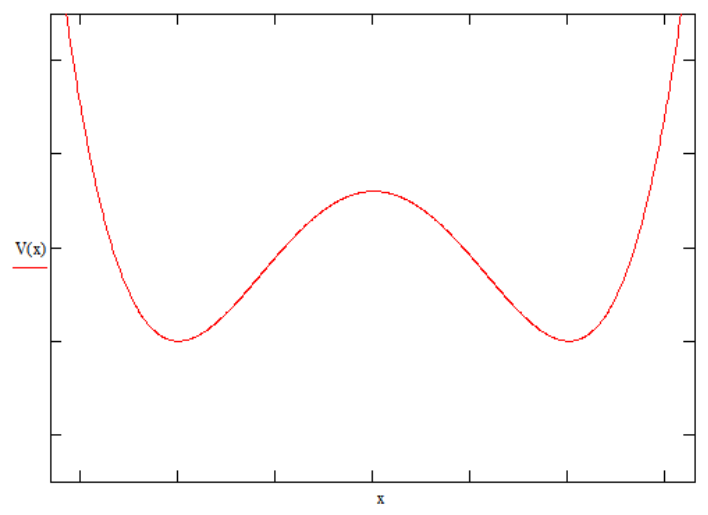

Figure 2: Potential $V(x)$ with mirror symmetry

The states "at rest on the left" and "at rest on the right" break the symmetry of the situation and, for this reason, they cannot be eigenstates of the Hamiltonian.

Given the potential $V(x)$, the Schrödinger equation independent of time can be solved to find the eigenfunctions $\left|\omega_{n}\right\rangle$ of the Hamiltonian. If the wavefunctions of the ground state $\left|\omega_{0}\right\rangle$ and of the first excited state $\left|\omega_{1}\right\rangle$ are graphed, the results of Figure 3 are obtained:
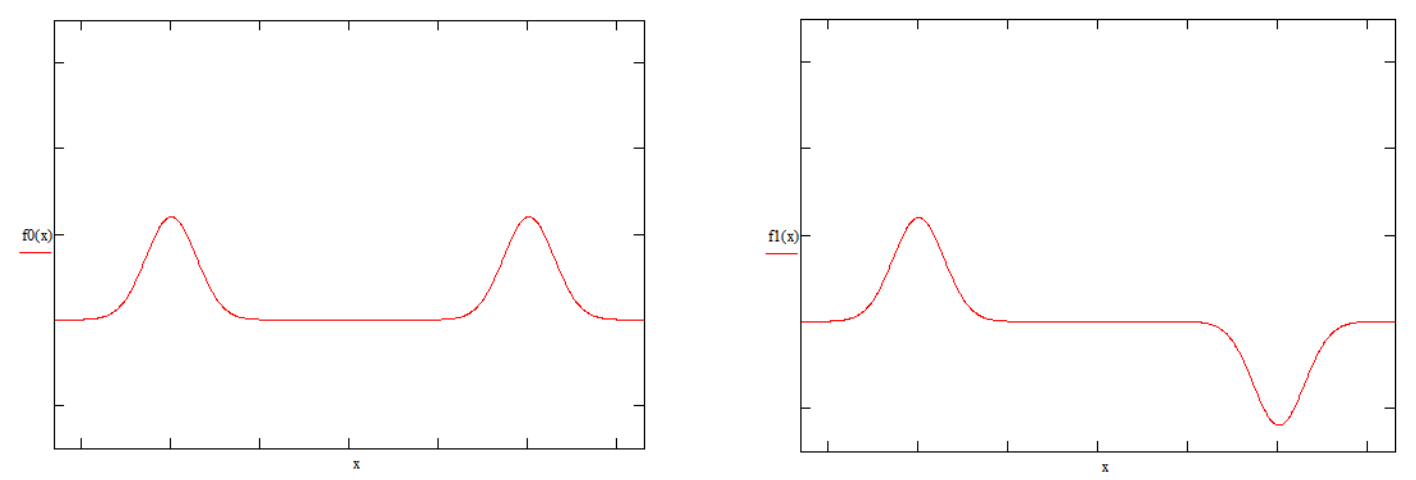

Figure 3. Form of the eigenfuncions of a particle subject to the potential $V(x)$. On the left, the ground state, symmetric under space reflections. On the righ, the first excited state, antisymmetric under space reflections. 
Figure 3 clearly shows that the eigenfunctions of the Hamiltonian preserve parity, that is, they are symmetric or anti-symmetric (as the expressions (6) and (7) of the original problem). These eigenstates do not correspond to states localized on the left or on the right, since both occupy both "wells."

Experimentally it is possible to place a quantum particle in one of the two minima of the potential, for instance, on the left. It is clear that this particle is not in an eigenstate of the Hamiltonian, but in a superposition. The left state $|L\rangle$ and the right state $|R\rangle$, represented by the expressions (8), correspond to wavefunctions as those shown in Figure 4.
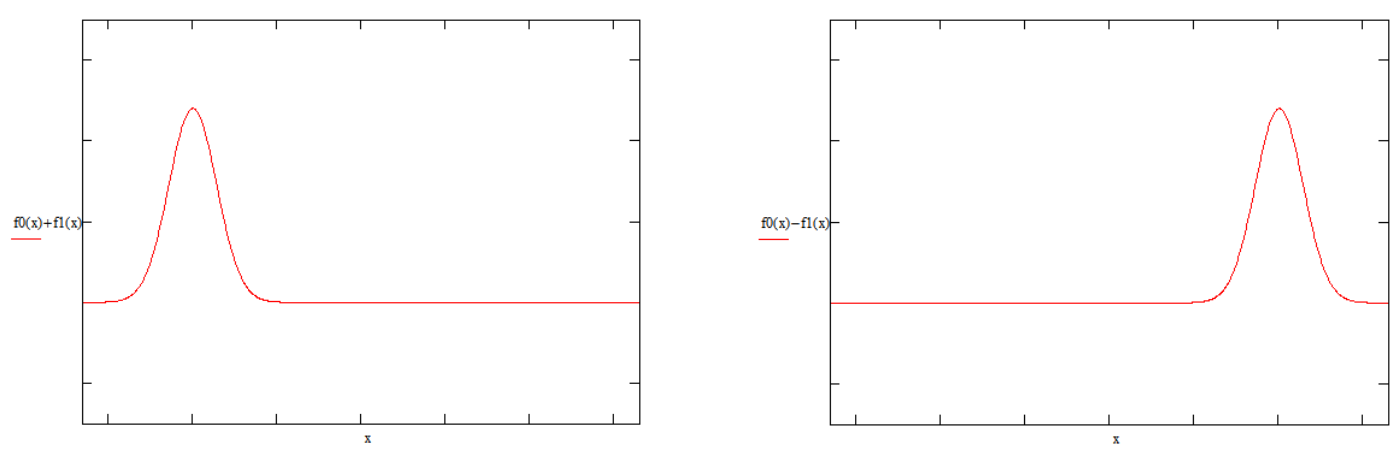

Figure 4. Form of the eigenfuncions of a particle in the states $|L\rangle$ and $|R\rangle$. On the left, the state $|L\rangle$ corresponds to a particle located on the left side. On the right, the state $|R\rangle$ corresponds to a particle located in the right side.

According to Hund, the fact that the states $|L\rangle$ and $|R\rangle$ cannot be stable is manifested by the quantum tunnel effect. Due to the dynamics imposed by Schrödinger equation, a particle in the state $|L\rangle$ has non-zero probability of passing to state $|R\rangle$. This probability can be computed as:

$$
P_{L \rightarrow R}=\left\langle R\left|e^{-i \frac{\hat{H}}{\hbar} t}\right| L\right\rangle=\left|\sin \left(\frac{\omega_{1}-\omega_{0}}{2 \hbar} t\right)\right|^{2}
$$

This expression says that, after a tunnel-time $t_{L \rightarrow R}=2 \pi \hbar /\left(\omega_{1}-\omega_{2}\right)$, the particle will have passed to the other side with certainty, and after a time $2 t_{L \rightarrow R}$, with certainty it will have returned to the original side.

Hund's idea is that the behavior of chiral isomers is analogous to that just described. Given the symmetry of the Coulombian Hamiltonian, the states $|L\rangle$ and $|R\rangle$ cannot be stable states of 
the molecule. Once it is admitted that, for some reason, certain molecules are not in their ground states, it is necessary to study the dynamics of such molecules. In the case of optical isomers, this would lead to accept that the molecules pass continuously from the state $|L\rangle$ to the state $|R\rangle$ and vice versa. Hund's view relies on admitting this behavior, but with the addition of certain restrictions. If the possibility of transmutation of isomers is accepted, then racemization can be explained; but for that explanation be acceptable, it is necessary that the tunnel-time $t_{L \rightarrow R}$ be extremely long, in such a way that the probability of transition be non-zero but very low during a long time. In this way, racemization turns out to be a process slow enough to be macroscopically observed.

In their article "On the time dependence of optical activity", Robert Harris and Leo Stodolsky (1981) face the problem of chiral isomers and emphasize the limitations of Hund's proposal. First, since chiral states are not eigenstates of the Hamiltonian, it is necessary to admit the existence of an exceptional kind of molecules that do not remain in their ground states. On the other hand, the requirement that the tunnel-time $t_{L \rightarrow R}$ be extremely long imposes a strong condition on the difference of energies $\omega_{1}-\omega_{2}$ between the ground state and the first excited state (see expression (9)): this difference must be extremely small. According to Harris and Stodolsky, this condition may be eventually fulfilled in very small molecules, but in general does not hold in most real systems.

For these authors, the key to solve both difficulties is the interaction between molecules, and they modify the Hamiltonian of the system with the purpose to describe such interaction. The central idea is that the paradox arises when the molecule is considered in isolation, whereas a real system actually consists of many molecules in interaction. For this reason, they propose to study the molecules in collision to each other: intermolecular collisions modify the dynamics of the whole composite system, and makes possible to express the tunnel-time $t_{L \rightarrow R}$, now conceived as a decay time, in terms of the parameters that define the collisions.

The proposal of Harris and Stodolsky is flexible enough to compute the decay time $t_{L \rightarrow R}$ in very different situations. But the point to stress here is that the solution to Hund's paradox offered by the authors is based on considering the interaction of the molecule with its environment, in this case composed of other molecules. This idea would be retaken soon later by the theory of decoherence. 


\section{5.- The appeal to decoherence}

In the field of quantum mechanics, one of the most serious interpretive problems is the so-called problem of quantum measurement, which consists in explaining how measuring devices record definite values of the observables (properties) of the measured quantum system when the system is in a superposition of the eigenstates of those observables. Hund's paradox can be conceived as a particular case of quantum measurement.

The usual formalism of quantum chemistry considers the molecule under study in its ground state, that is, in the lowest energy eigenstate $\left|\omega_{0}\right\rangle$ of the Hamiltonian. On the other hand, each observable of the molecule is represented by a mathematical operator. According to quantum mechanics, in order to compute the probability of measuring a certain value of an observable, it is necessary to express the state of the molecule in the basis of the eigenstates of the operator representing the observable to be measured: the eigenvalues corresponding to those eigenstates represent the possible definite values of the observable. In our case, the observable of interest is chirality, represented by the operator $\hat{Q}$ with eigenstates $|L\rangle$ and $|R\rangle$. As explained in the previous section, the ground state of the molecule, expressed in the chiral basis, is:

$$
\left|\omega_{0}\right\rangle=\frac{1}{\sqrt{2}}(|L\rangle+|R\rangle)
$$

In this case, the state of the molecule is a superposition of $|L\rangle$ and $|R\rangle$. In Schrödinger words: the cat is neither alive nor dead; he is in a superposition of both states. However, the result of a measurement is always $|L\rangle$ or $|R\rangle$. The question is then the same as in the case of the measurement problem: how to explain that we measure definite values of chirality when the state of the system is a superposition of $|L\rangle$ and $|R\rangle$. In this case, the problem is to account for the transition from the superposition to one of the chiral states, say, $|L\rangle$ :

$$
\left|\omega_{0}\right\rangle=\frac{1}{\sqrt{2}}(|L\rangle+|R\rangle) \rightarrow|L\rangle
$$

In the orthodox interpretation of quantum mechanics, this transition is simply postulated: when measured, the system "collapses" to one of the possible states. Since the final state is no longer a superposition, it is concluded that the measured observable acquires a definite value. This postulate, formulated for the first time by Werner Heisenberg in his famous article of 1927, is known as "the collapse postulate" (Heisenberg 1927). Collapse is an indeterministic process: 
due to its form (see expression (10)), $\left|\omega_{0}\right\rangle$ has a probability of 0.5 of collapsing to the state $|L\rangle$ and a probability of 0.5 of collapsing to the state $|R\rangle$. If many individual measurements are performed on identical systems with the same initial conditions, it is possible to define an ensemble, analogously to classical statistical mechanics (Ballentine 1998): the state of the ensemble after collapse can be represented by means of a density operator:

$$
\hat{\rho}_{\text {measured }}=\frac{1}{2}|L\rangle\left\langle L\left|+\frac{1}{2}\right| R\right\rangle\langle R|
$$

The density operator $\hat{\rho}_{\text {measured }}$ is interpreted as representing a mixed state: a statistical mixing of the states $|L\rangle$ and $|R\rangle$ of the individual members of the ensemble, that is, a state with the same structure of classical mixed states. This means that this mixed state can be interpreted as a measure of ignorance: in each measurement, the individually measured member of the ensemble is actually in one of the states $|L\rangle$ or $|R\rangle$, but the observer cannot predict in which particular state it is.

Although simple, the hypothesis of collapse gives rise to several difficulties. Besides to being an ad hoc hypothesis that duplicates the kinds of quantum evolutions, it does not explain why and when collapse occurs. Moreover, collapse seems to introduce a kind of action-at-adistance when applied to entangled states of spatially separated particles, such as in the case of EPR-type experiments. For these reasons, alternative solutions to the measurement problem have been proposed. During the last decades, in the field of physics the measurement problem is being faced in the light of the theory of environment induced decoherence (EID). This program has been developed by the group led by Wojciech H. Zurek (1981, 1982, 1991, 1993, 2003) and based in the Los Alamos National Laboratory.

The EID program relies on the study of the effects of the interaction between a quantum system, considered as an open system, and its environment. As some authors claim (e.g., see Leggett 1987, Bub 1997), decoherence has become the "new orthodoxy" in the physical community. Nowadays, the theory of decoherence is studied in many fields such as atomic physics, quantum optics and condensed matter. In particular, it has acquired a high relevance in the domain of quantum computation, in which the phenomenon of decoherence represents the main obstacle to the implementation of hardware that takes advantage of superpositions to process information. 
In its orthodox version, the EID theory of decoherence is an approach that applies to open systems since, as its name shows, it considers the system under study $S$ embedded in an environment $E$ that induces decoherence. The composite system $U=S \cup E$ is a closed system that evolves according the Schrödinger equation and whose initial state is the tensorial product of the initial states of its components: ${ }^{2}$

$$
\hat{\rho}_{U}=\hat{\rho}_{S} \otimes \hat{\rho}_{E}
$$

Since the Schrödinger equation is unitary, $\hat{\rho}_{U}(t)$ never approaches a final state that could be interpreted classically. However, if the partial trace on the evolved total state $\hat{\rho}_{U}(t)$ is computed, the degrees of freedom of the environment $E$ are removed and the reduced state of the subsystem $S$ of interest is obtained:

$$
\hat{\rho}_{S}(t)=\operatorname{Tr}_{E}\left(\hat{\rho}_{U}(t)\right)
$$

This reduced state, computed by ignoring the degrees of freedom of the environment, is not governed by the Schrödinger equation, but evolves according a non-unitary master equation, different in each particular case. Therefore, the dynamics of the reduced state can lead to a final stable state. For this reason, according to the EID approach, the study of decoherence is based on the analysis of the evolution of the reduced state, represented as an operator expressed in a certain basis. Either by explicitly calculating the state or by analyzing the master equation case by case, it is possible to determine if, under certain conditions, the reduced state operator becomes diagonal, that is, loses the interference terms that preclude classicality.

In many models of physical systems, in which the number of degrees of freedom of the environment is huge, it can be proved that, after a time $t_{D}$ called "decoherence time', the reduced state $\hat{\rho}_{S}(t)$ becomes diagonal in a basis representing the classically behaving observable. In the usual explanations of the phenomenon it is said that such diagonalization is the manifestation of the process of decoherence induced by an environment with a large number of degrees of freedom. In other words, the continuous "monitoring" exerted by the environment on the system produces the "degradation" of the quantum states of the system in diagonal states which, for this reason, represent a classical situation (Paz and Zurek 2002): "the environment distills the

\footnotetext{
2 The theory of decoherence works with the representation of the quantum state in the von NeumannLiouville space. The ket $|\varphi\rangle$ is represented in this space by an operator $\hat{\rho}=|\varphi\rangle\langle\varphi|$. The advantage of this space is that more general states can be represented in it (see Landau and Lifshitz 1958).
} 
classical essence of a quantum system." (Zurek 2003, p. 3). This amounts to conceiving the operator $\hat{\rho}_{S}(t)$ as the physical-mathematical object that represents the state of a subsystem of the whole closed system, and to supposing that that subsystem became classical.

In the Editorial 37 of the journal Foundations of Chemistry, its editor Eric Scerri (2011) explicitly considers the problem of isomerism, in particular, of optical isomerism; his purpose is to confront the opinion according to which this problem is a symptom of the inability of quantum mechanics to account for the chemical structure of a molecule. With a sharp observation, Scerri relates the problem of isomerism with the measurement problem in quantum mechanics, and states that the question about molecular structure "is part of a bigger problem that has long plagued the foundations of quantum mechanics, namely the problem of the collapse of the wavefunction. [...] this problem has gradually begun to dissolve with the growing realization of the role of quantum decoherence in physics and other disciplines." (Scerri 2011, p. 4). On the basis of the extensive literature on decoherence, Scerri claims that the problem of optical isomerism was dissolved with the interaction of the molecule with its environment: "The study of decoherence has shown that it is not just observations that serve to collapse the superpositions in the quantum mechanics. The collapse can also be brought about by molecules interacting with their environment." (Scerri 2011, p. 4; for a similar claim, see Scerri 2013).

These statements clearly show that Scerri is well acquainted with the recent developments in physical-chemistry. However, it is necessary to consider also the conceptual analysis of the explanations given by the theory of decoherence, coming from philosophy. This task may lead to conclusions different from those widespread in the chemistry and the physics communities.

\section{6.- Decoherence, chirality and interpretation}

In Section 4, when Hund's paradox was introduced, the focus was directed on the isolated molecule. According to the EID approach, this simplification is the result of an inadequate idealization. A real molecule is an object exposed to the interaction with the very large number of atoms and other molecules that form its environment. Then, a less idealized model consists in considering also the quantum states of the environment. In this model, given a chiral molecule, the initial state of the composite system molecule+environment is: 


$$
\left|\varphi_{S E}\right\rangle=\left|\omega_{0}\right\rangle \otimes\left|\varepsilon_{0}\right\rangle=\frac{1}{\sqrt{2}}|L\rangle \otimes\left|\varepsilon_{0}\right\rangle+\frac{1}{\sqrt{2}}|R\rangle \otimes\left|\varepsilon_{0}\right\rangle
$$

where $\left|\varepsilon_{0}\right\rangle$ is the initial state of the environment. Following the usual arguments of the theory of decoherence (see Schlosshauer 2007), it is supposed that the $\left|\varepsilon_{j}\right\rangle$ are the states of the environment $E$, and that there is a particular Hamiltonian that describes the interaction between the system and the environment. This interaction produces two important effects:

- The states of the two systems become correlated:

$$
\begin{aligned}
& |L\rangle \otimes\left|\varepsilon_{0}\right\rangle \rightarrow|L\rangle \otimes\left|\varepsilon_{L}\right\rangle \\
& |R\rangle \otimes\left|\varepsilon_{0}\right\rangle \rightarrow|R\rangle \otimes\left|\varepsilon_{R}\right\rangle
\end{aligned}
$$

- The states of the environment become rapidly (approximately) orthogonal:

$$
\left\langle\varepsilon_{L} \mid \varepsilon_{R}\right\rangle \rightarrow 0
$$

The theory of decoherence presupposes that the interaction is capable of producing these two effects, which lead the state of the whole system to become:

$$
\left|\varphi_{S E}\right\rangle \rightarrow\left|\psi_{S E}\right\rangle=\frac{1}{\sqrt{2}}|L\rangle \otimes\left|\varepsilon_{L}\right\rangle+\frac{1}{\sqrt{2}}|R\rangle \otimes\left|\varepsilon_{R}\right\rangle
$$

Since the evolution of the whole closed system is unitary, its state $\left|\psi_{S E}\right\rangle$ cannot approach a final classical-like state. In other words, the state operator

$$
\hat{\rho}_{S E}=\left|\psi_{S E}\right\rangle\left\langle\psi_{S E}\right|
$$

always includes interference terms. However, by taking the partial trace of the environmental degrees of freedom on the state operator of the whole system, the reduced state of the molecule is obtained, in which the degrees of freedom of the environment vanished due to the orthogonality of the environmental states (see expression (17)):

$$
\hat{\rho}_{S}=\operatorname{Tr}_{E}\left(\hat{\rho}_{S E}\right)=\frac{1}{2}|L\rangle\left\langle L\left|+\frac{1}{2}\right| R\right\rangle\langle R|
$$

Because of the similarity between this reduced state $\hat{\rho}_{S}$ and the classical mixed state $\hat{\rho}_{\text {measured }}$ of the expression (12), the theorists of the EID approach consider that $\hat{\rho}_{S}$ denotes a mixed state containing only the representation of classical correlations and, as a consequence, it can be interpreted in terms of ignorance. According to their view, the system is in one of the states $|L\rangle$ 
or $|R\rangle$, and probabilities measure our ignorance about the definite state of the system. In this way, the theory of decoherence would have solved the problem underlying Hund's paradox.

In spite of the wide application of the decoherence program, the ability of the theory to solve the traditional measurement problem has been largely discussed, and serious doubts have been raised by means of different arguments (Healey 1995, Bacciagaluppi 2012). Let us recall that the collapse hypothesis establishes that the state of the system becomes instantaneously and indeterministically one of the states of the superposition and, as a consequence, the system acquires a well-defined value for the measured observable. The case of decoherence is completely different: here there is no collapse; as Zurek himself points out, the state "appears to have collapsed" (Zurek 1981, p. 1517). As a consequence, it cannot be concluded that the system acquires a well-defined value for the measured observable. In fact, the state $\left|\psi_{S E}\right\rangle$ is a superposition at any time: the superposition never disappears through a unitary evolution. For this reason, although the reduced state operator $\hat{\rho}_{S}$ lacks interference terms, this does not allow us to suppose that what is observed at the end of the process is one of two definite events: either the event associated with $|L\rangle$, or the event associated with $|R\rangle$. On this basis, the theoretical physicist Stephen Adler concludes: "I do not believe that either detailed theoretical calculations or recent experimental results show that decoherence has resolved the difficulties associated with quantum measurement theory" (Adler 2003, p. 136).

The criticism of the philosopher specialized in quantum mechanics Jeffrey Bub (1997) is even stronger: according to him, stating that what is observed at the end of the measurement process is a definite event not only is unjustified, but also contradicts some basic precepts of the theory. In fact, reading $\hat{\rho}_{S}$ as a classical statistical mixture amounts to interpreting the involved probabilities as measures of the ignorance about the well-defined underlying state that determines the precise value of the measured observable. But this stance is inconsistent with a standard assumption in quantum mechanics, the eigenstate-eigenvalue link, according to which a quantum observable has a definite value if and only if the state of the system is an eigenstate of this observable. In our case, without an effective collapse, the state $\hat{\rho}_{S E}$ of expression (19) is always a superposition of the states $|L\rangle \otimes\left|\varepsilon_{L}\right\rangle$ and $|R\rangle \otimes\left|\varepsilon_{R}\right\rangle$ : neither the system $S$ is in one of the states $|L\rangle$ or $|R\rangle$, nor the environment $E$ is in one of the states $\left|\varepsilon_{L}\right\rangle$ or $\left|\varepsilon_{R}\right\rangle$. Therefore, according to the eigenstate-eigenvalue link, even if decoherence occurs, the system $S$ cannot have a definite value of chirality. In other words, the reduced density operator $\hat{\rho}_{S}$ not only is unable to account 
for the occurrence of a single event associated with a definite value of chirality, but the assumption of that occurrence is inconsistent with the eigenstate-eigenvalue link.

Another criticism against the supposed solution to the measurement problem via decoherence is based on the difference between the state of the closed system and the reduced state assigned to the open system. Already in 1966, Bernard d'Espagnat established the difference between a proper mixture -the mixed state of a closed system- and an improper mixture -the state of an open system, obtained by tracing off the degrees of freedom of its environment-. According to d'Espagnat (see also 1976), although proper and improper mixtures are represented by the same mathematical object - a density operator-, they represent different concepts: improper mixtures cannot be interpreted in terms of ignorance. For instance, in his well-known book about decoherence, Maximilian Schlosshauer claims that: "Since the two systems $A$ and $B$ are entangled and the total composite system is still described by the superposition, it follows from the standard rules of quantum mechanics that no individual definite state can be attributed to either one of the subsystems. Reduced density matrices of entangled subsystems therefore represent improper mixtures" (Schlosshauer 2007, p. 48). This means that, strictly speaking, a reduced density operator is only "a calculational tool" for computing expectation values (Schlosshauer 2007, p. 48). For this reason, the author warns us "against a misinterpretation of reduced density matrices as describing a proper mixture of states" (2007, p. 69).

Perhaps the fact that proper and improper mixtures cannot be distinguished from a mathematical viewpoint is what leads many authors to suppose that the reduced state $\hat{\rho}_{S}$ is the quantum state of the subsystem $S$ in the same sense as $\hat{\rho}_{S E}$ is the quantum state of the whole closed system $S \cup E$. However, that mathematical feature essentially depends on the formalism used to express the theory, in particular, the Hilbert space formalism. But the situation might be different in other cases. For instance, it has been proved (Masillo, Scolarici and Sozzo 2009) that proper and improper mixtures are represented by different density operators in the so-called quaternionic formulation of quantum mechanics; ${ }^{3}$ hence they can be distinguished also from a mathematical viewpoint. Moreover, this mathematical representation is compatible with their different time-evolutions as represented in the orthodox Hilbert space formalism.

\footnotetext{
${ }^{3}$ The quaternionic formulation of quantum mechanics is a formalism based on quaternion fields instead of complex fields (see Adler 1995).
} 
The argumentation for the difference between quantum states (proper mixtures) and reduced states (improper mixtures) was further developed in a recent paper by Sebastian Fortin and Olimpia Lombardi (2014). The authors show that reduced states obtained by partial traces are not the quantum states of the subsystems of a closed system. In particular, it is proved that a reduced state is a kind of coarse-grained state of the composite system to which it belongs, and for this reason it cancels the correlations between the subsystem and other subsystems with which it interacts or is entangled.

These and other arguments have led certain physicists, even some whose contributions were central in the development of the program of decoherence, to manifest their skepticism about the relevance of decoherence regarding the measurement problem. For instance, Erich Joos explicitly says: "Does decoherence solve the measurement problem? Clearly not." (Joos 2000, p. 14). In a recent article, Scerri (2012) recognizes that the question of whether decoherence explains quantum measurement is a subtle matter, and refers to the review paper of Guido Bacciagaluppi (2012); in this paper the author points out that, although naive claims of the kind that decoherence gives a complete answer to the measurement problem are still somewhat part of the "folklore" of the matter, decoherence as such does not provide a solution to the measurement problem, at least not unless it is combined with an appropriate interpretation of the theory.

The above considerations lead us to conclude that, once the theory of decoherence is analyzed from a conceptual perspective, the appeal to the phenomenon of decoherence by itself does not seem to be a promising way to solve Hund's paradox. If chiral states are not eigenstates of the Hamiltonian and each molecule is in a superposition of both states, decoherence does not make such superposition to disappear and, as a consequence, there is no adequate answer yet to the question about why chiral molecules have a definite chirality.

\section{7.- Conclusions}

Since the beginnings of quantum mechanics in the 1920s, chemistry and physics have converged at a common point: quantum chemistry. This domain has been the seat of the stronger debates about the relationship between the theories coming from the two disciplines. In this paper we have addressed the problem of optical isomerism and the challenges that it implies regarding the idea that chemistry, at the molecular level, can be explained by quantum mechanics. In this 
context, we have formulated with precision Hund's paradox and the solution proposed by its own author, pointing out the difficulties of such proposal. This has led us to consider in detail the currently most appealed solution, based on the phenomenon of decoherence. However, the conceptual analysis of the scope of the theory of decoherence has allowed us to conclude that the appeal to the diagonalization of the reduced state of the molecule in interaction with its environment is not sufficient to explain the definite value of chirality observed in laboratory.

On the basis of these results it is possible to put forward the idea that, if the links between molecular chemistry and quantum mechanics are to be strengthened, it is necessary to adopt some interpretation of quantum mechanics that supplies an acceptable answer to the measurement problem and, derivatively, explains why certain observables adopt a definite value even in superposition states. Among the realist non-collapse interpretations, the Modal-Hamiltonian Interpretation (Lombardi and Castagnino 2008) seems particularly suitable for this purpose, because its selects the Hamiltonian as the observable that always acquires a definite value and that governs the selection of the remaining definite-valued observables of the system. This interpretation, besides being compatible with the EID program (Lombardi, Fortin, Castagnino and Ardenghi 2011), is in perfect resonance with molecular chemistry, in the context of which molecules are almost always described in their stationary states, that is, in eigenstates of the Hamiltonian. The modal-Hamiltonian perspective would explain the definite value of chirality if this observable commutes with the Hamiltonian of the whole system in which the chiral molecule is immersed. Nevertheless, this proposal is beyond the limits of the present article and will be the topic of a future work.

Acknowledgements: We are very grateful to Eric Scerri for our discussions about the problem of isomerism. This publication was made possible through the support of grant 57919 from the John Templeton Foundation.

\section{8.- References}

Adler, S. L. (1995). Quaternionic Quantum Mechanics and Quantum Fields. New York: Oxford University Press. 
Adler, S. (2003). "Why decoherence has not solved the measurement problem: A response to P. W. Anderson." Studies in History and Philosophy of Modern Physics, 34: 135-142.

Bacciagaluppi, G. (2012). "The role of decoherence in quantum mechanics.” In E. N. Zalta (ed.), The Stanford Encyclopedia of Philosophy (Winter 2012 Edition), URL = <http://plato.stanford.edu/ archives/win2012/entries/qm-decoherence/>.

Bader, R. (1994). Atoms in Molecules: A Quantum Theory. Oxford: Oxford University Press.

Ballentine, L. (1998). Quantum Mechanics: A Modern Development. Singapore: World Scientific.

Berlin, Y. A., Burin, A. L. and Goldanskii, V. V. (1996). "The Hund paradox and stabilization of molecular chiral states." Zeitschrift für Physik D, 37: 333-339.

Bub, J. (1997). Interpreting the Quantum World. Cambridge: Cambridge University Press.

d'Espagnat, B. (1966). “An elementary note about mixtures.” In A. De-Shalit, H. Feshbach and L. van Hove (eds.), Preludes in Theoretical Physics. Amsterdam: North-Holland.

d'Espagnat, B. (1976). Conceptual Foundations of Quantum Mechanics. Reading MA: Benjamin.

Dirac, P. A. M. (1929). "Quantum mechanics of many-electron systems." Proceedings of the Royal Society of London A, 123: 714-33.

Fortin, S. and Lombardi, O. (2014). "Partial traces in decoherence and in interpretation: What do reduced states refer to?” Foundations of Physics, 44: 426-446.

Harris, R. A. and Stodolsky, L (1981). "Time dependence of optical activity." The Journal of Chemical Physics, 74: 2145-2155.

Healey, R. A. (1995). "Dissipating the quantum measurement problem.” Topoi, 14: 55-65.

Heisenberg, W. (1927). "Über den anschaulichen Inhalt der quantentheoretischer Kinematic und Mechanik." Zeitschrift für Physik, 43: 172-198. English version: (1983). "The physical content of quantum kinematics and mechanics". In J. A. Wheeler and W. H. Zurek (eds.), Quantum Theory and Measurement. Princeton: Princeton University Press. 
Hendry, R. F. (2004). "The physicists, the chemists, and the pragmatics of explanation." Philosophy of Science, 71: 1048-59.

Hendry, R. F. (2008). "Two conceptions of the chemical bond." Philosophy of Science, 75: 909920.

Hendry, R. F. (2010). “Ontological reduction and molecular structure.” Studies in History and Philosophy of Modern Physics, 41: 183-191.

Hettema, H. (2009). "Explanation and theory foundation in quantum chemistry." Foundatios of Chemistry, 11: 145-174.

Hettema, H. (2012). Reducing Chemistry to Physics. Limits, Models, Consecuences. Groningen: University of Groningen.

Hund. F. (1927). “Zur Deutung der Molekelspektren. III.” Zeitschrift für Physik, 43: 805-826.

Joos, E. (2000). "Elements of environmental decoherence.” In P. Blanchard, D. Giulini, E. Joos, C. Kiefer and I.-O. Stamatescu (eds.), Decoherence: Theoretical, Experimental, and Conceptual Problems, Lecture Notes in Physics, Vol. 538. Heidelberg-Berlin: Springer.

Landau, L. D. and Lifshitz, E. M. (1958). Quantum Mechanics: Non-Relativistic Theory. Oxford: Pergamon Press.

Leggett, A. J. (1987). "Reflections on the quantum measurement paradox.” In B. J. Hiley and F. D. Peat (eds.), Quantum Implications. London: Routledge and Kegan Paul.

Lombardi, O and Castagnino, M. (2008). "A modal-Hamiltonian interpretation of quantum mechanics." Studies in History and Philosophy of Modern Physics, 39, 380-443.

Lombardi, O. and Castagnino, M. (2010). "Matters are not so clear on the physical side." Foundations of Chemistry, 12: 159-166.

Lombardi, O., Fortin, S., Castagnino M. and Ardenghi, J. S. (2011). "Compatibility between environment-induced decoherence and the modal-Hamiltonian interpretation of quantum mechanics.” Philosophy of Science, 78: 1024-1036.

Masillo, F., Scolarici, G. and Sozzo, S. (2009). "Proper versus improper mixtures: towards a quaternionic quantum mechanics." Theoretical and Mathematical Physics, 160: 1006-1013. 
Nagel, E. (1961). The Structure of Science. New York: Harcourt, Brace \& World.

Paz, J. P. and Zurek, W. H. (2002). "Environment-induced decoherence and the transition from quantum to classical.” In D. Heiss (ed.), Fundamentals of Quantum Information, Lecture Notes in Physics, Vol. 587. Heidelberg-Berlin: Springer (page numbers are taken from arXiv:quant-ph/0010011).

Scerri, E. R. (2011). "Editorial 37.” Foundations of Chemistry, 13: 1-7.

Scerri, E. R. (2012). “Top-down causation regarding the chemistry-physics interface: a sceptical view." Interface Focus, 2: 20-25.

Scerri, E. R. (2013). "Philosophy of chemistry: where has it been and where is it going." In J.-P. Llored (ed.), The Philosophy of Chemistry: Practices, Methodologies, and Concepts. Newcastle: Cambridge Scholars Publishing.

Schlosshauer, M. (2007). Decoherence and the Quantum-to-Classical Transition. Berlin: Springer.

Sutcliffe, B. T. and Wolley, R. G. (2011). "A comment on Editorial 37." Foundations of Chemistry, 13: 93-95.

Sutcliffe, B. T. and Wolley, R. G. (2012). "Atoms and molecules in classical chemistry and quantum mechanics.” In R. F. Hendry and A Woody (eds), Handbook of Philosophy of Science. Vol. 6, Philosophy of Chemistry. Oxford: Elsevier.

Szabo, A and Ostlund, N. S. (1996). Modern Quantum Chemistry: Introduction to Advanced Electronic Structure Theory. Mineola NY: Dover.

Wolley, R. G. (1976). "Quantum theory and molecular structure”. Advances in Physics, 25: 2752.

Wolley, R. G. (1978). “Must a molecule have a shape?”. Journal of the American Chemical Society, 100: 1073-1078.

Wooley, R. G. (1982). "Natural Optical Activity and the Molecular Hypothesis". Structure and Bonding, 52: 1-35.

Wolley, R. G. (1998). “Is there a quantum definition of a molecule?”. Journal of Mathematical Chemistry, 23: 3-12. 
Zurek, W. H. (1981) "Pointer basis of quantum apparatus: into what mixture does the wave packet collapse?”. Physical Review D, 24: 1516-1525.

Zurek, W. H. (1982). "Environment-induced superselection rules”. Physical Review D, 26: 18621880.

Zurek, W. H. (1991). "Decoherence and the transition from quantum to classical". Physics Today, 44: 36-44.

Zurek, W. H. (1993). "Preferred states, predictability, classicality and the environment-induced decoherence". Progress of Theoretical Physics, 89: 281-312.

Zurek, W. H. (2003). "Decoherence, einselection, and the quantum origins of the classical". Reviews of Modern Physics, 75: 715-776 (page numbers are taken from arXiv:quant$\mathrm{ph} / 0105127)$. 